\title{
A MULTILAYER-ROUTING-STRATEGY WITH DYNAMIC LINK RESOURCE ADAPTATION
}

\author{
Robert Prinz ${ }^{1}$, Dr. Andreas Iselt ${ }^{2}$ \\ ${ }^{1}$ TU-Muenchen, Institute for Communication Networks, Arcisstr 21, 80333 Munich, \\ prinz@lkn.tum.de \\ ${ }_{2}^{2}$ Siemens AG, Corporate Technology, Information and Communication, Otto-Hahn-Ring 6, \\ 81730 Munich, Germany, andreas.iselt@siemens.com
}

Abstract: With the automation in optical networks based on the introduction of a control plane (GMPLS, ASON) it will be possible to adapt the transport network dynamically to the traffic requirements of packet based client layers. This allows reducing leased line costs and improving the throughput. In some cases the network throughput limitation comes from the limited switching capacity of transit routers. This is especially the case when Multiservicenetwork elements are used, which usually are implemented as an extension of SDH network elements with packet switching capabilities. Hereby the packet switching matrix usually only covers a fraction of the whole interface capacity of the node, since the main switching is expected to be carried out in the TDM (SDH) domain. An intelligent Multilayer-Routing-Strategy can relief the packet layer by circumventing packet switching with transport shortcuts. In this paper we present such a new Multilayer-Routing-Strategy, that allows to route demand with a distributed routing mechanism. This is complemented by a centralized optimization instance, that optimizes the overall network status. Simulative investigations allow a first quantification of the advantage of this approach.

\section{INTRODUCTION}

The introduction of automated connection control in optical transport networks using ASON (Automatically Switched Optical Networks) [3] or GMPLS (Generalised Multi-Protocol Label Switching) [4] together with the standardisation of interfaces like UNI (User Network Interface) and NNI (Network Network 
Interface) will allow establishing connections immediately on customer demand. If the "customer" is a network operator (e.g. IP/MPLS network operator, ISP) he can adapt the capacity of his network to the actual load pattern. It is also possible that this network operator does not even own the transport network infrastructure, but leases it dynamically based on the online offer of competing suppliers.

The challenge is to automate the bandwidth adaptation process of the client network with the target of a stable, cost-efficient and dynamic network, that optimally distributes the load and can react on resource constraints. The Multilayer-Routing-Strategy (MLRS) that is presented here is described using a MPLS network (client layer) on an optical network (server layer), but is also applicable to other network technologies.

\section{ARCHITECTURE}

In Figure 1 the regarded network architecture is depicted. A two-layer network with packet switched MPLS over circuit switched (optical) transport is assumed. The elements of the client and the server layer are collocated at some positions. This may be realized by integration of packet switching and circuit switching capabilities, as it is often found in Multiservice-Nodes.

We further assume that an User-Network-Interface (UNI) between the layers is available, that allows the client layer to request the setup and the release of connections from the server layer. This mode of operation is usually called Overlay Model. The principle of operation is that the links between nodes in the client layer, which are realised as connections in the underlying server layer are adapted dynamically in accordance to the requirements (i.e. traffic demand) of the client layer.

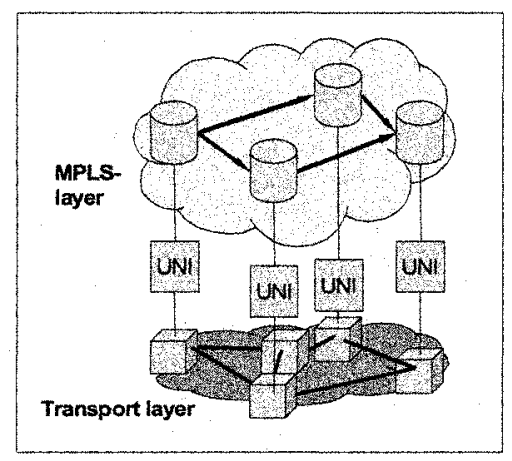

Figure 1. Network architecture

In the following sections a strategy for this adaptation is proposed and evaluated. 


\section{MULTILAYER ROUTING STRATEGY}

We developed a multilayer routing strategy (see also [1]) which is divided in two separate processes. The first process is a distributed algorithm which is implemented in all routers of the client network. It is responsible for routing new connections in the client network. This process can add new link resources (optical channels which are dynamically requested from the transport network) to the existing client network in reaction to a capacity bottleneck if necessary. The distributed algorithm is faster and more reliable than a central management solution. The second process which has a lower priority runs on one or more central servers and tries to reoptimize the current routing and link capacity adjustment on specific events with the objective to minimize the transport cost. This process is not time critical and even in case of a server failure network operation is not impaired.

The following assumptions are considered:

- The client network uses MPLS and all MPLS paths have a reserved bit rate.

- The available capacity of links and routers is distributed via OSPF-TE in the whole network. Each node knows the currently available network resources.

- Source routing is used and could be done by RSVP-TE using the Explicit Route Object.

- Each node has a transit connection data base which matches each transit connection to its originating node. When using RSVP-TE for MPLS path setup its data base can be used.

- The central servers for the reoptimization have access to all connection data of the client network.

\subsection{The Algorithm for new Connection Requests}

If a new client layer connection request arrives at node $S$ with the maximum bit rate $d_{S, T}$ between source node $S$ and target node $T$, the algorithm for a new connection request is applied, which distinguishes four different cases:

Case 1: There is a path $p$ that has enough free capacity on each involved element (source-, target- and transit-routers as well as the links of the path $p$ ). The connection will use this path $p$. If there is more than one possible path, the connection will use the best path (e.g. the path with the lowest hop count and with the most available capacity). This is illustrated in Figure 2(a).

Case 2: Source node $S$ and target node $T$ have enough free capacity but there is no path with sufficient free capacity between them.

Now the client network can add a new link to its topology or increase the bit rate on an existing link by creating a new optical channel by the UNI to resolve the capacity bottleneck in the network. The problem of the selection of the node pair to 
be connected by a new optical channel is solved by optimization. The objective is to find a cost minimized solution with the greatest benefit for the current network. The condition which has to be met here is, that a path $p$ is generated which has enough free capacity on all involved elements for the current connection request (see Figure 2(b)).
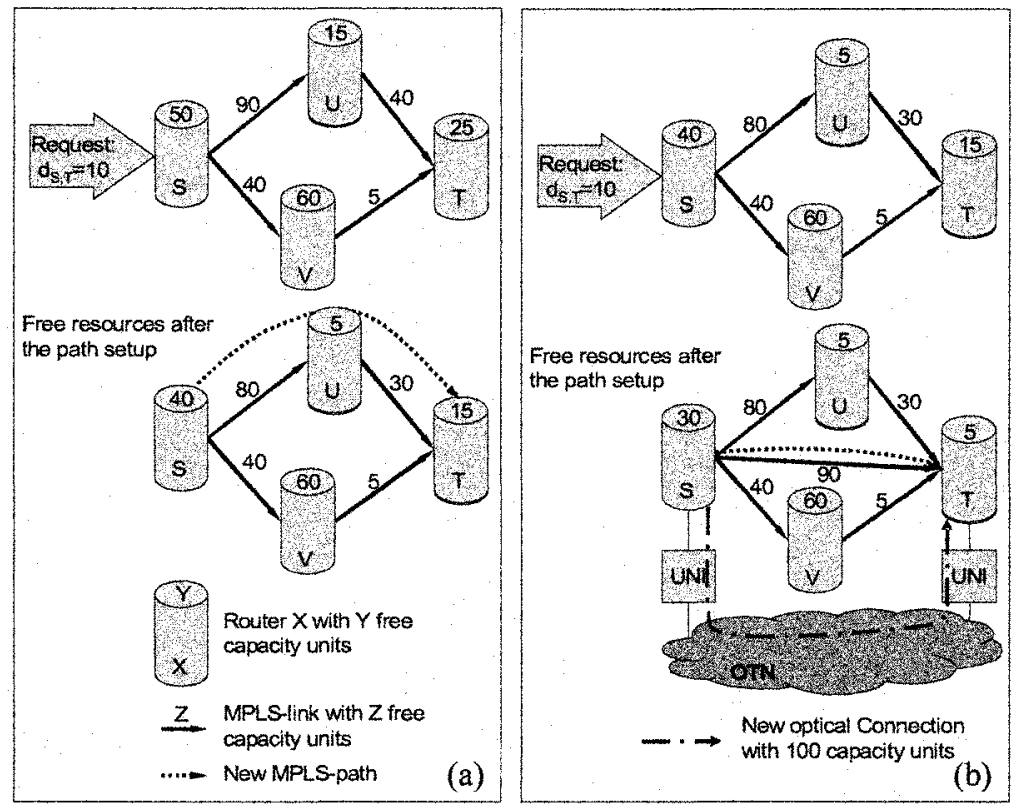

Figure 2. New Connection request and the resulting routing in case 1 (a) and in case 2 (b)

Case 3: At least one of the nodes $S$ or $T$ has not sufficient free capacity and it is not possible to free capacity on this router by rerouting existing connections (e.g. all existing connections on this highly loaded router begin or end at it). The new connection request must be blocked (see Figure 3(a)).

Case 4: Source node $S$ or target node $T$ or both have not enough free capacity, but it exists one or more transit connections on each highly loaded router with (in sum) more than $d_{S, T}$ bit rate usage. Now this router signals the originating node of selected transit connections (e.g. the transit connections with the greatest bit rate reservation) to find an alternative path for these connections without using this router. Provided that there is enough capacity available in the optical layer, it must be possible to reroute the connection. For this rerouting also a new link can be added if necessary. After the rerouting procedure the connection request can be routed through the network, possibly adding a new channel to the network as described in case 2 (see Figure 3(b)). 
To case 3 and 4: If target node $T$ has not enough resources for the new connection request source node $S$ can not decide whether the target node can solve this problem by rerouting or not. So node $S$ has to signal an inquiry to target node $T$ (see in Figure 3(a) and (b)).
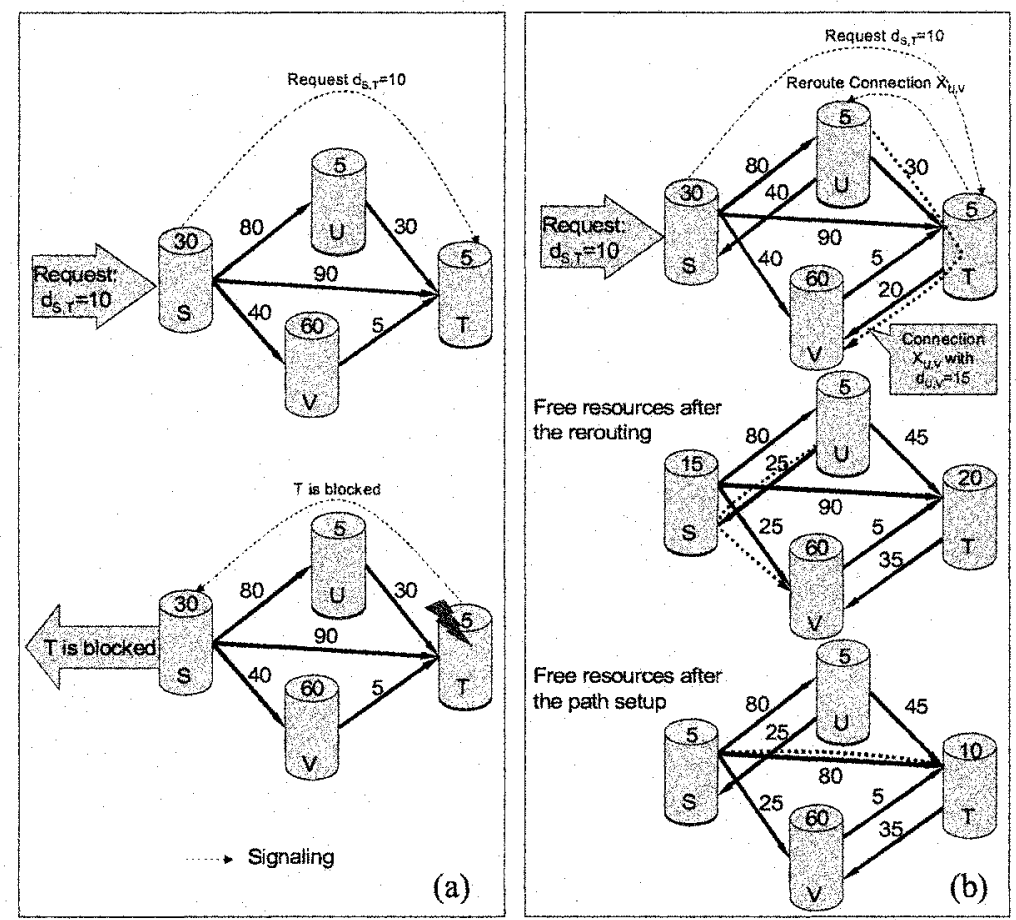

Figure 3. New Connection request and the resulting routing in case 3 (a) and in case 4 (b)

\subsection{The Reoptimization Process}

In addition to the algorithm for a new connection request described in chapter 3.1 a second mechanism with a lower priority is involved. It tries to reoptimize the routing with the objective to free and release optical channels. It may be executed periodically or triggered by a connection release. Besides the required constraints for this optimization other constraints like limiting the maximum number of reroutable connections per iteration are imaginable. The reoptimisation process runs on one or more synchronized central servers and has access to all necessary data for the reoptimization (e.g. connection data and leased link data). A reoptimization step is shown in Figure 4. 


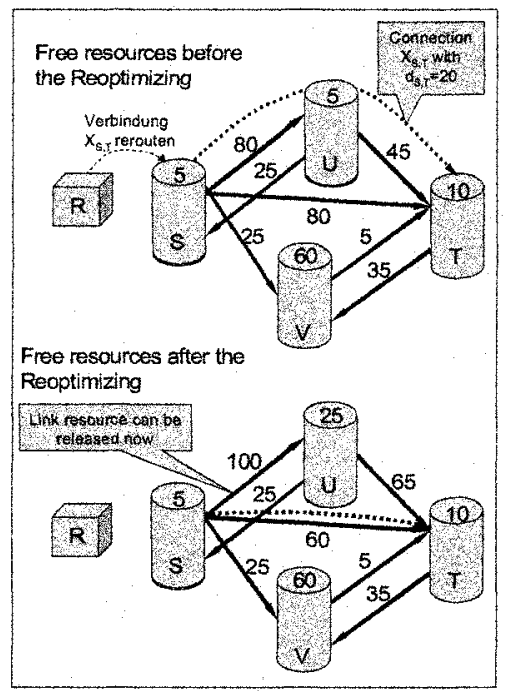

Figure 4. Network resources before and after reoptimization

\section{SIMULATION}

We developed a simulator for the multilayer routing strategy described above to analyze the dynamic behavior of the algorithm. To compare our strategy using the dynamic link resource management to a single layer strategy with static network resources we modified the mechanism described above in that way, that for the static case no new resources can be added to the existing topology.

\subsection{Simulation Conditions}

For the simulation we use a pan-European network from [2] which is displayed in Figure 5. The used bidirectional traffic intensity matrix from [2] is shown in Table 1 together with a bidirectional link capacity adjustment optimized for this traffic in the static case (each link capacity must be a multiple of 10 capacity units). Also the capacity $N C$ of each network node is optimized and must be a multiple of 40 capacity units (see in Table 1). The capacity of the nodes is used for the dynamic case as well as the static case. In the dynamic case the network has no links at the beginning of the simulation and the algorithm can add new channels with the granularity of 10 capacity units from any node to any other node at any time always with the same cost.

The connection requests are generated randomly. The mean value $i_{S, T}$ of the neg. exponentially distributed inter arrival time of the node pair $S-T$ is given by the equation (1). Thereby $s$ is the mean value of the also neg. exponentially distributed 
service time, which has the fixed value of 30 time units in our simulations. $A_{S, T}$ is the traffic intensity value of node pair $S-T$ out of Table 1 . To simulate different network loads the traffic intensity $A_{S, T}$ is multiplied with a filling ration $f$, which we vary in a range of 0.1 to 0.4 in 0.05 steps. The filling ratios between 0.1 and 0.4 lead to values between 1419 and 5828 connection requests within the 500 time units simulation period.

$$
i_{S, T}=\frac{s}{A_{S, T} \cdot f}
$$

Every 10 time units the network is reoptimized by the described mechanism of section 3.2. The path for each connection is restricted to a maximum of two transit nodes and the capacity of each connection has a fixed value of one capacity unit.

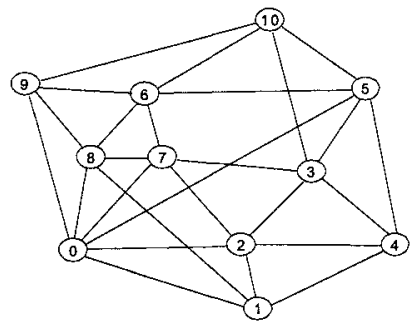

Figure 5. COST239 Network

Table 1. Bidirectional traffic matrix, node capacities $N C$ and bidirectional link capacities

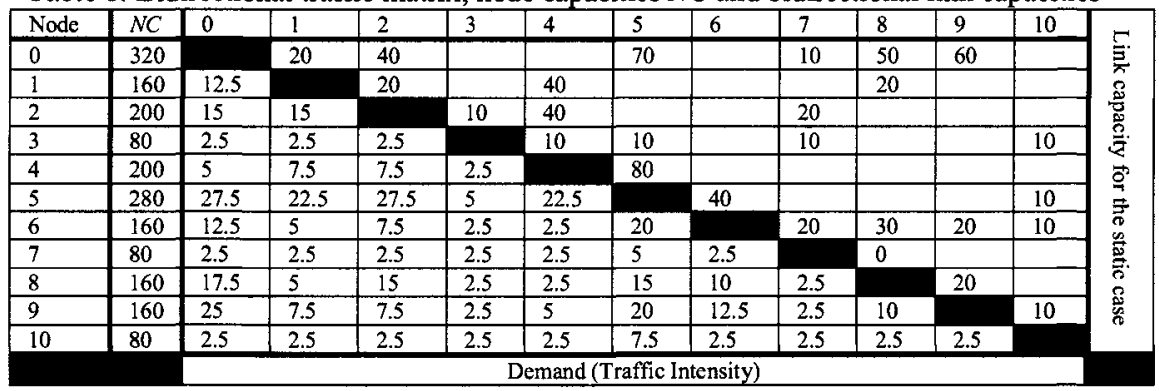

\subsection{Results}

Figure 6 shows the results as a function of the filling ratios $f$. In the static case the blocking ratio $b$ is zero up to the filling ratio of $f=0.25$. It can be seen that the blocking in the static case is higher than in the dynamic case where even for a filling ration of $f=0.3$ no connection is blocked. Regarding the resource requirements it can be seen, that in the dynamic case the network requests links in the simulation period which rises from 126 links for $f=0.1$ up to 377 links for $f=0.4$ in a nearly linear manner. It is remarkable that the mean link capacity $Ø L C$ of the 
network in the dynamic case lies between $13 \%$ for $f=0.1$ and $35 \%$ for $f=0.4$ of the link capacity used in the static case, which has a value of 1360 capacity units.

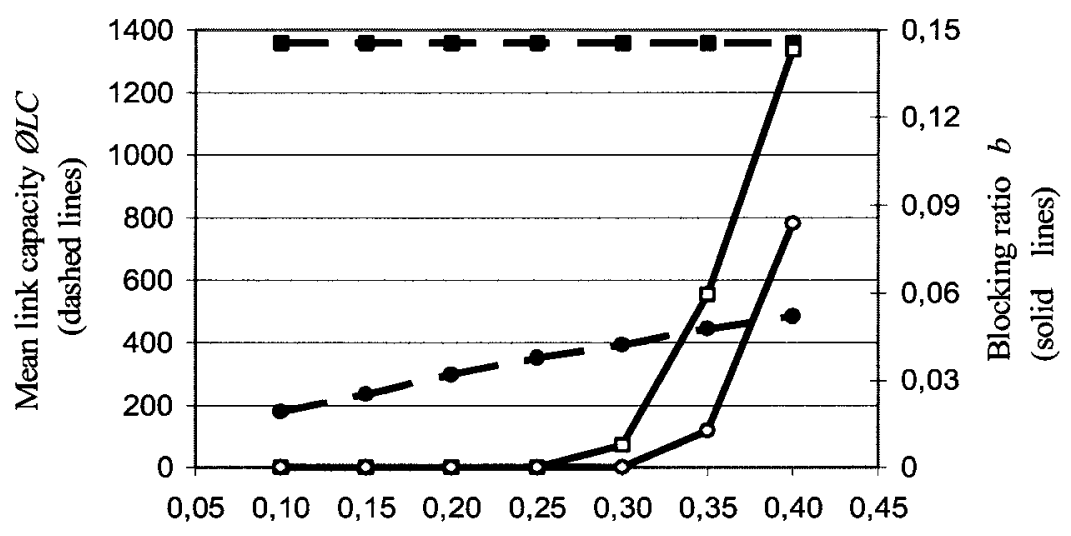

Filling ratio $f$

Figure 6. Simulated mean link capacity $\emptyset L C$ (dashed lines) and blocking ratio b (solid lines)

\section{SUMMARY}

In this paper we have presented a multilayer routing strategy that enables dynamic adaptation of link resources in the client network. This dynamic adaptation to changing demands bears potential for saving cost (required link capacities and switching matrix sizes) and improving blocking. The strategy has been investigated by simulation. The simulation results show that the resource requirements (mean link capacities) can be reduced remarkably. Additionally the blocking probabilities can be improved allowing a higher network utilization without experiencing higher blocking ratios.

\section{REFERENCES}

[1] R. Prinz, A. Iselt, "Eine Multilayer-Routing Strategie für GMPLS-Netze mit dynamischer Linkressourcenanpassung," 5. ITG-Fachtagung Photonische Netze, Leipzig, 2004, pp. 53-57.

[2] P. Batchelor et al., "Ultra High capacity optical transmission networks: Final report of action COST239," in Faculty of Electrical Engineering and Computing, Zagreb, 1999.

[3] ITU-T Rec. G8080/Y.1304, "Architecture for the Automatically switched Optical Network (ASON)," November 2001

[4] E. Mannie, D. Papadimitriou et al., "Generalized MPLS Architecture," Information Draft, draft-ietf-ccamp-gmpls-architecture-01.txt, November 2001 